Praxis International Journal of Social Science and Literature

Impact Factor: SJIF $2020=5.754$ ISSN: 2581-6675

Volume - 4, Issue - 2, February - 2021

Website: www.pijssl.com, E-mail: editor.pijssl@gmail.com

\title{
मेधा पाटकर और विस्थापितों के प्रति उनकी प्रतिबद्धता
}

\author{
औरजीना मैरी लाकाडोंग \\ असिस्टेंट प्रोफेसर, जानकी देवी मेमोरियल कॉलेज, \\ दिल्ली विश्वविद्यालय, नई दिल्ली
}

\section{सारांश}

'नर्मदा बचाओ आंदोलन' पर बहुत कुछ लिखा जा चुका है और बोला गया है। लेकिन इस शोध-पत्र के माध्यम से मैं जिस मुख्य तथ्य को रेखांकित करना चाहती हूँ वह है कि इस आंदोलन के फलस्वरूप नर्मदा घाटी में आए सामाजिक-राजनीतिक बदलाव-शिक्षा, महिलाओं की स्थिति, समाज में मौजूद कठोर जातीय दिवारों का गिरना, राजनीतिक अधिकारों के प्रति सजगता और भ्रष्ट नेता और सरकारी अफसरों का विरोध। अगर नर्मदा बचाओं आंदोलन या मेधा पाटकर नहीं होती तो राजनीतिक पार्टिया लोगों को बहकाकर अपना स्वार्थ सिद्ध करती। शायद कुछ लोगों को मुआवज़ा भी मिल जाता पर अधिकांश लोग अव्यवस्था में खो जाते या छूट जाते, जैसे बारगी और हरसौद गाँवों के लोगों के साथ हुआ। अगर ये संघर्ष नहीं होता तो 1990-91 तक बाँध बन गया होता और लोग कुछ नहीं कर पाते।

बीज शब्द: नर्मदा बचाओ आंदोलन, विकास की संकल्पना, बुनियादी-अधिकार, संसाधन, नव-निर्माण, औद्योगीकरण, जीवन-मूल्य, अहिंसा, जन-आंदोलन, जल-नियोजन, दलित, आदिवासी, समाजवाद, संवैधानिक मूल्य, पुनर्वास, छूआछूत, जातीय समानता, त्री-सशक्तिकरण।

\section{प्रस्तावना}

मेधा पाटकर ने लगभगकई साल नर्मदा घाटी में बिताए है और ज्यादात्तर वही रहती है, वहाँ के स्थानीय लोग भूल चुके है कि वे कभी मुम्बई से उनके बीच आई थी। वे नर्मदा से जुड़े हर आंदोलन में 1985 से ही सक्रिय रही हैं। साथ ही जहाँ भी जल, जमीन, जंगल की बात होती है और लोगों के साथ अन्याय होता है, वे उपेक्षितों के साथ खड़ी दिखाई देती है। इस बात का प्रखर उदाहरण वर्तमान किसान आंदोलन है। टिकरी बॉर्डर से किसान आंदोलन को संबोधित करते हुए मेधा पाटकर ने खेती किसानी संघर्ष में महिलाओं के महत्व को स्वीकारने पर ज़ोर दिया। वर्तमान सरकार द्वारा किसानों के खिलाफ
लाए तत्कालीन तीनों कृषि कानून - कृषक उपज व्यापार और वाणिज्य विधेयक (संवर्धन और सरलीकरण)-2020, कृषि कीमत अश्वासन और (सशक्तिकरण और संरक्षण) कृषि सेवा करार विधेयक- 2020, आवश्यक वस्तु संशोधन विधेयक-20201 के संदर्भ में उन्होंने कहा कि "तीनों कृषि कानून चंद पूंजीपतियों के पोषण के लिए लाए गए हैं। यह कानून लागू किए गए तो राशनव्यवस्था तबाह हो जाएगी मजदूरों और किसानों के लिए घर की रसोई को चलाना असंभव हो जाएगा। यह तीनों कानून वापस नहीं हुए तो सबसे अधिक नुकसान किसान मजदूरों का होगा।"2 मेधा पाटकर इसके अलावा पश्चिम बंगाल में नंदीग्राम, महाराष्ट्र में पूना के आसपास और आंध्रप्रदेश में 
काकीनाड़ा, जैसे स्थानों में सेज विरोधी आंदोलन से भी जुड़ी हुई थी।

पीड़ितों के हक में आवाज उठाने के लिए जब भी, जहाँ भी मौका मिलता है, वे संगठनों से जुड़कर, जनब आदोलन में सक्रिय रूप से भाग लेती है। मुम्बई की गरी बस्तियों को जबउजाड़ा जा रहा था, तब मेधा जी के नेतृत्व में तीन संगठनों 'घर बचाओं, घर बनाओ' 'कैरीवाला संगठन' व 'राष्ट्रीय शहर विकास संघर्ष' के माध्यम से सरकार के विरूद्ध संघर्ष जारी किया गया। गहन गाँधीवादी विचारधारा से प्रभावित मेधा पाटकर ने सरदार सरोवर परियोजना से प्रभावित होने वाले लगभग 37 हज़ार के गाँवों के गरीब विस्थापित,आदिवासी, दलित, किसान, मजदूर के हक के लिए संघर्ष के शस्त्र'सत्याग्रह' और 'अहिंसा' को चुना। कई ऐसे मौके आए हैं जब लोगों ने मेधा पाटकर का जुझारू रूप देखा है। उन्होंने मध्य प्रदेश, गुजरात और महाराष्ट्र की सरकार के अलावा विश्व बैंक से भी लड़ाई ठानी है। कई बार डूबते गाँवों के साथ डूबकर मर जाने की धमकी देने वाली मेधा पाटकर शायद ही कभी पीछे हटी हो। 1991 में 22 दिनों का अनशन करके वे लगभग मौत के मुँह में चली गई थी। 1993 और 1994 में भी वे ऐसे लंबे उपवास कर चुकी हैं।मेधा पाटकर का मानना है कि विकास के बदले में चुकाई जाने वाली मानवीय कीमत, विकास नहीं होती। वे कहती है -"विकास की परिभाषा किसी भी भाषा, किसी भी माध्यम में आप ढूढ़े तो यही होती है कि संसाधनों का उपयोग करके जो जीने के, जो जीविका के बुनियादी अधिकार हैं, या जरूरते हैं, उसकी पूर्ति करना। कोई भी विकास विरोधी नहीं है, न हम हैं, न सरकार भी हो सकती है। लेकिन सरकार एक प्रकार का विकास नज़रिया ये कहते हुए जब सामने लाती है कि उन संसाधनों के आधार पर वो लाभ ही लाभ पाएँगें आर्थिक या भौतिक) न उसमें जिन लोगों के हाथ में पीढ़ियों से। लेकि(लाभ संसाधन हैं, उनका न कोई योगदान होगा नियोजन में, न ही उनको लाभों में भागीदारी या हिस्सा मिलेगा। तब
जाकर हमको अपने विकास की संकल्पना भी रखने का अधिकार है।“3

वस्तुतः मेधा पाटकर का संघर्ष,तोड़ने की नही बल्कि नवनिर्माणकी बात करता है। उनकी विचारधारा विकास और औद्योगीकरण के खिलाफ नहीं है। पीड़ितों के हक में आवाज़ उठाने का मतलब विकास विरोधी नहीं होता। जो योजना सरकार द्वारा बनाई जाती है, वो मुट्ठी भर लोगों के लाभ के लिए बनाई जाती है। 'सिंगूर' में नैनों परियोजना, मानसरोवर बांध, आदि इसका उदाहरण है। भारत कृषि प्रधान देश है,यहाँ पर कृषि आधारित उद्योग अधिक होने चाहिए ताकि अधिसंख्य लोग लाभान्वित हो सके। जब ऐसा नहीं होता, तभी जन आंदोलन से जुड़े लोग विरोध करने को विवश होते हैं। आस्ट्रेलिया को देखे तो देश का पूरा बीच का क्षेत्रफल रेगिस्तान है, लेकिन फिर भी सरकार ने किसानों को ध्यान में रखते हुए रेगिस्तान के कुछ भाग को कृषियोग्य भूमि बनाने की परियोजना बनाई है,जिससे सरकार और जनता दोनो लाभान्वित हुई है। इस तरह हमारी सरकार भी किसानों, गरीबों, विस्थापितों को ध्यान में रखते हुए योजना बनाएगी तो विरोध की कोई गुंजाइश नहीं रहेगी।

सरकार और पूँजीपतियों के खिलाफ इस संघर्ष में मेधा जी ने कभी अपनी बुनियादी जीवन मूल्यों को नहीं भुलाया है। वे संघर्ष को निर्माण से जोड़ने की बात करती है। उनके संघर्ष की नींव अहिंसा पर टिकी हुई है। इसीलिए वे सशस्त्र संघर्ष का समर्थन नहीं करती। उन्हें पता है कि सशस्त्र संघर्ष दूरगामी विचारधारा को मंजिल तक नहीं पहुंचा सकता। सशस्त्र संघर्ष शांति, क्षमता और न्याय के साथसाथ जीने और जीने के आधार को छीनलेती है। मेधा पाटकर के लिए अहिंसा केवल रणनीति नहीं है, एक मूल्य है। जीने का अधिकार, और जीवन प्रणाली के लिए ही यह संघर्ष चल रहा है। आम जनता तो अपनी रोजीरोटी-, अपना स्वास्थ्यशिक्षा-, अपनी प्रकृति और जीने के अधिकार को सुरक्षित करने के लिए तमाम जनआंदोलन चला रही है। अगर सरकार उन तक पहुँचेगी, 
उनका सम्मान करेगी, उनको उनका स्थान देगी, उनसे संवाद करेगी तो वे सरकार से जरूर जुड़ेगे।

मेधा पाटकर अपने संघर्ष के द्वारा मुख्य तथ्यों को रेखांकित करना चाहती है और पूछती है "आज इस देश मे बहुत सारी जमीन, पानी, जंगल, खनिज, संपदा, भूजल होते हुए भी $80 \%$ लोग अपनी बुनियादी जरूरतों को पूरा नहीं कर पा रही हैऔर चंद लोग लक्षाधीश, कब्जाधीश बन रहे हैं, तो ऐसा क्यों है? कारर्पोरेट वर्ल्ड और सेज़ के नाम पर जो कंपनियों को बड़ीबड़ी जमीनों के हिस्से देने की साज़िश चल रही है और सबसे बड़ी बात की कंपनियों को छूट देते हुए किसानों को बाकी सब्सिडी भी नहीं देना, जो बेबस किसानों को आत्महत्या के लिए मजबूर करता है। इस प्रकार की स्थितियाँ समाज में विरोधाभास पैदा करती है, इसलिए जहाँजहाँ सेज़ थोपा जा रहा है, चाहे आंध्रप्रदेश मे काकीनाड़ा हो, चाहे महाराष्ट्र में रायगढ़ हो, चाहे पश्चिम बंगाल का नंदीग्राम हो या झारखंड, उड़ीसा में पॉस्कों जैसी परियोजना हो, लोग विरोध में खड़े हो रहें और कहींकहीं रोकथाम हो चुकी है।" 4

ये तमाम देशीविदेशी कंपनियाँ-, हमारे देश में व्याप्त प्राकृतिक संसाधान और मनुष्य के श्रमों के आधार पर ही अपना लाभ, अपनी तिजोरी भरना संभव कर पा रहें हैं और लोगों के हाथ में जो पुराने पीढ़ियों से धरोहर जैसे प्राकृतिक संसाधन हैं, उनको हस्तांतरित किया जा रहा है। वस्तुतः यह दोनों स्थितियाँ, सरकार की आंदोलन से निपटने की इस तरह की नीतियाँ जनतंत्र को खतरे में धकेल रही है।

मेधा पाटकर मानती है कि इन तमाम समस्याओं का हल निकाला जा सकता है अगर सरकार गरीबों, किसानों, विस्थापितों को ध्यान में रखकर अपनी नीतियाँ बनाए। इस संबंध में मेधा पाटकर कहती है कि -"हमलोग जल नियोजन चाहते हैं तो विकेन्द्रित जल नियोजन, ताकि हरेक को अपने नज़दीक से नज़दीक जल स्रोत से अपनी
प्यास बुझाने का मौका मिले। हम औद्योगीकरण चाहते हैं तो खेती खत्म करके उद्योग नहींजो खाली जमीन पड़ी है, उसमें उद्योग लग जाए और उद्योग से पर्यावरणीय प्रदूषण या प्राकृतिक संसाधनों का विनाश और बड़े पैमाने पर विस्थापन न हो। 5

उनका मानना है कि अगर सरकार गाँवगाँव-, शहर की बस्तीनियोजन का अधिकार लेकर बस्ती तकजाएगी और जो नियोजन होगा, उसमें पहले अधिकार उन्ही का होगा तो फिर समस्या ही खत्म हो जाएगी। तभी रोज़गार, समाज, का निर्माण करने वाला विकास होगा, न कि रोज़गार खत्म करने वाला। वो रोज़गार खेतीऔर उद्योग की बीच की दूरी मिटाने वाला होगा। जहाँ महिलाएँ, बच्चे ही नहीं दलितआदिवासी-, मजदूरकिसान सभी, जो आज अबला या दुर्बल घटक माने जातेहैं, उनको पहली प्राथमिकता मिलेगी। इसी आधार पर इस देश में समता, न्याय, समाजवाद जो संवैधानिक मूल्य है, उनकी स्थापना हो पाएगी।

\section{संघर्ष के फलस्वरूप नर्मदा घाटी के समाज में आए परिवर्तन}

उत्पीड़ितों और विस्थापितों के लिए समर्पित मेधा पाटकर का जीवन, शक्तिऊर्जा और वैचारिक उदात्तता की जीती जागती मिसाल है। पिछलेकई वर्षों से उनके संघर्षशील जीवन ने उन्हें पूरे विश्व के सबसे महत्वपूर्ण शाख्सियतों में शुमार किया है। अपने दशकों के इस संघर्ष में मेधा पाटकर ने तमाम परियोजनाओं से विस्थापित, किसानों, आदिवासियों के लिए न्यायोचित और टिकाऊ विकास, न्याय और उचित पुनर्वास की माँग की है।

घाटी के स्थानीय लोगों के लिए जीवन, एक संघर्ष कर दिया गया है और संघर्ष ही जीवन बन गया है। उनकी साहस की कहानियाँ, प्रतिबद्धता, बलिदान और आकांक्षाओं को मेधा पाटकर का प्रतिनिधित्व मिला। पाटकर का यह संघर्ष गरीबकिसानों-, दलितों, आदिवासियों में अपने अधिकारों के प्रति जागरूकता तो 
लाया ही, साथ ही उनके समाज के रूढ़िगत व्यवस्थाओं, परंपराओं को भी तोड़ने में सक्षम हुआ है। एक ओर जहाँ त्रियों ने पर्दा प्रथा को तो त्यागा इसके साथ ही घर के चारदिवारी से निकलकर सरकार के अन्यायअत्याचार और भ्रप्टाचार के खिलाफ आवाज़ उठाने के लिए वे सड़क पर निकल कर आई। दूसरी ओर समाज में व्याप्त मंदिरमस्जिद का साम्प्रदायिक भेद, कठोर जातीय व्यवस्था को इस संघर्ष ने तोड़ा, जिससे सारी दीवार मिट गई।

यहाँ के स्थानीय लोगों के लिए जीवन भर का यह संघर्ष, जीवन का पाठ बन गया है। मेधा पाटकर को नर्मदा घाटी के वासी दीदी, ताई या जीजी कहकर सम्बोधित करते हैं। मेधा पाटकर के प्रयासों को स्थानीय लोगों ने समझा है, उसे सराहा है और हमेशा उनका सम्मान किया है। विस्थापना को झेलने वाला पुनया वासव मेधा पाटकर के संबंध में कहते है -'अगर मेधा ताई और अन्य आंदोलनकर्त्ता हमारे घाटी न आए होते तो हमारी जातिय समुदाय का क्या होता? मेधा ताई से पहले भी गुजरात से कई अन्य संगठनों के लोग आए थे परन्तु वे सिर्फ गुजरातीबोलते थे। वे कभी भी संगठित आम बैठक आयोजित नहीं करते थे बल्कि व्यक्तिगत स्तर पर गाँव के नेताओं को समझाने की कोशिश करते थे। दूसरी ओर मेधा ताई ये सुनिश्चित करती है कि जो भी बैठक में आया है, उसे भाग लेने का मौका दिया जाए। जिसमें बच्चे, औरते और बूढ़े भी शामिल होते हैं। तब हमने समझा और पहचाना कि इस संघर्ष के लिए ताई सबसे उचित व्यक्ति है। वे बाकी कार्यकताओं के साथ गाँवगाँव घूमती है। उन्होंने यहाँ की स्थानीय भाषा पावरी और भिलाली भी सीखी। वे हमारे साथ, हमारे बीच रहती है और हमारे सामाजिक और पारिवारिक उत्सवों में भाग लेती है। हमारे जीवनशैली को समझने की कोशिश करती है।ताई ... ने अपनी जिन्दगी इस संघर्ष में लगा दी। उन्होंने नर्मदा बचाओं आंदोलन का प्रतिनिधित्व किया, हमें लड़ने क लिए प्रेरणा दी और हमें आश्वस्त किया। हम दृष्टिहीन थे पर ताई के पास दूरदृष्टि थी।"6 वस्तुतः नर्मदा बचाओं आंदोलन न केवल जल, पर्यावरण, विस्थापन की संघर्ष
बयान करता है बल्कि घाटीसमाज में पड़ने वाले सामाजिक भूमिका को भी रेखांकित करता है। इस आंदोलन ने लोगों को जागरूक और सशक्त बनाया है। घाटी और आस बच्चा अपने गाँव-पास के गाँवों का बच्चाको डूब से बचाने, सरकार से जमीन के बदले जमीन की मांग रखने और आंदोलन द्वारा चलाए हर रैली, धरना, जुलूस में भाग लेना, उनके लिए रोजमर्रा की बात हो गई है।

नर्मदा बचाओं आंदोलन की अद्भूत संघर्ष नीतियाँ है। गाँधीवादी विचारों को केन्द्र में रखते हुए सूदूर क्षेत्रों के लोगों को जहाँ संगठित किया वहीं दूसरी ओर इस इच्छाशक्ति के साथ कि न सिर्फ प्रभावित लोग बल्कि देश के दूसरे जनआंदोलन भी उनसे जुड़ सके। इसलिए यह आंदोलन स्वतंत्रता के बाद उभरने वाले उन आंदोलनों में से एक है, जो आम नागरिकों को गहरे रूप में प्रभावित करता है और प्रेरणा स्र्रोत बन गया है। घाटी के कई ऐसे स्थानीय कार्यकर्ता है जिन्होंने इस आंदोलन को व्यापक और प्रभावी बनाने में अपनी सारी जिन्दगी लगा दी। इन स्थानीय लोगों ने संगठित रूप से एक होकर सरकार की नीतियों का विरोध किया। घाटी के हर गाँव से इस संघर्ष को सशक्त रूप देने के लिए एक स्थानीय आवाज़ मौजूद रहा है, जो अशिक्षित गरीब जनता को सरकार की अमानवीय नीतियों से परिचित कराता है और आने वाले दुष्प्रभाव को रेखांकित करता है।

पुनया वासव, गोकखरू मंगल, कैलाश अवसाया, शांताबाई मोकाटी, कमला यादव आदि ऐसे स्थानीय कार्यकर्ता है जिन्होंने इस आंदोलन को अपना जीवन बना लिया है। इस संघर्ष ने न सिर्फ बाँध परियोजना संबंधी समझ बल्कि जीवन के हर पक्ष को समझने और पहचानने की समझ भी पैदा की है। नर्मदा बचाओं आंदोलन ने उनके समाज, उनके परंपराओं, रीतिरिवाज को जहाँ सुरक्षित रखने की भरपूर कोशिश की है, वही समाज में मौजूद जड़विजड़ित थोती परंपराओं और रिवाजों को तोड़ने में भी मदद की है।

शुरू में स्थानीय लोगों ने मेधा पाटकर पर विश्वास नहीं किया, उन्हें यही लगा कि जरूर इसमें उनका 
निजी स्वार्थ छिपा हुआ है। मेथा पाटकर ऐसी बातों से परिचित थी, इसके बावजूद वे गाँवगाँव जाकर सभा बुलाती थी और लोगों से प्रश्न पूछती थी कि अगर गाँव डूब धीरे समय के साथ -धीरे .जाएगा तो आप कैसे जीएँगे स्थानीय लोग, मेधा पाटकर की बात को समझने लगे जब उन्होंने बाँध को बनते और आसपास के पर्यावरण को नष्ट होते हुए देखा।

शुरू में मेधा पाटकर महाराष्ट्र फिर मध्य प्रदेश के गाँवों में गई और बाँध के बनने से गाँव पर पड़ने वाले प्रभाव से लोगों को परिचित कराया, उनमें जागरूकता फैलाई। एक ओर मेधा पाटकर और कार्यकर्ताओं ने बाहरी दुनिया से इनका परिचत कराया तो दूसरी ओर आदिवासियों के दैनिक जीवन में भी कई परिवर्तन लेकर आई, विशेषकर आपसी लड़ाई में शांतिपूर्ण समाधान, शिक्षा, और कई अंधविश्वासपूर्ण अभ्यासों जैसेमहिला को 'डाकन' बुलाना आदि से मुक्ति दिलाई। शिक्षा और लोगों की उस तक पहुँच ने समाज के सोच में कई सकारात्मक परिणाम शिक्षा और लोगों की उस तक पहुँच ने समाज के सोच में कई सकारात्मक परिणाम दिखाए हैं-

\section{शिक्षा की व्यवस्था}

नर्मदा बचाओं आंदोलन महाराष्ट्र और मध्यप्रदेश के आदिवासी लोगों के बच्चों के लिए 'जीवनशाला' (1992) नामक विद्यालय चलाती है। जिसकी लगभग 13 शाखाएँ इन क्षेत्रों में फैली हुई है। विद्यालय खोलने की मांग लोगों के बीच से उठी, क्योंकि उन्हें इस बात का अहसास था कि एक बच्चे के जीवन की नींव शिक्षा ही होती है। कुछ गाँवों भादल, मनीबेली और जलसिंधी में सरकारी स्कूल विद्यमान थे, परन्तु फिर भी वहाँ की आधी से अधिक जनता अशिक्षित थी। क्योंकि उन स्कूलों में पढ़ाने के लिए कोई नहीं आता था। अगर आते तो भी नहीं पढ़ाते थे। क्योंकि स्कूल जंगलों के बीच में होते हैं तो वहाँ तक आने-जाने का कोई साधन नहीं होता। दूसरी तरफ अगर कोई ईमानदार शिक्षक पहुँच भी जाता तो उसे सरकारी कामों, जैसे सर्वे करना, स्वास्थ्य विभाग की सहायता, करना आदि में लगा दिया जाता था। अधिकांश
सरकारी स्कूल के ये अध्यापक 'स्वतंत्रता दिवस' या गणतंत्र दिवस के दिन ही स्कूल में अपनी हाजरी लगाते थे और कभी-कभी तो गाँव के मुखिया को दस रुपए देकर झण्डा फहरा देते थे। वहाँ सारे सरकारी काम कागजों पर होते हैं, RTI के सूचना के मुताबिक भादल गाँव (मध्य प्रदेश) में 1975 में स्कूल खोला गया और कागजी रिकार्ड बताते हैं कि स्कूल से कई विद्यार्थी पढ़कर निकले और पढ़कर अब बडवानी और बड़े-बड़े शहरों में नौकरी कर रहे हैं। सरकारी रिकार्ड इतने अच्छे से रखा गया है कि उसमें विद्याथियों के नाम, उनके बारे में और उन्हें दी जाने वाली सुविधाएँ- जैसे साबुन, किताबें, तेल आदि का वर्णन है। ये विद्यालय महज सरकारी फाईलों में दर्ज है। मेधा पाटकर कहती है “हमने पाया कि कागज पर स्कूल थे लेकिन एक भी कार्यात्मक स्कूल जमीन पर मौजूद नहीं था।"7

फलस्वरूप आंदोलन ने गाँवों में 'जीवनशाला'चलाया और आज यह कार्यक्रम सफलतापूर्वक अपना काम कर रहे हैं। गाँव के बच्चे जीवनशाला में शिक्षा ग्रहण करते हैं, जहाँ उन्हें शिक्षा के साथ-साथ उनके अधिकारों के बारे में, आंदोलन के संघर्ष के बारे में शिक्षित किया जाता है। यह विद्यालय परंपरागत शिक्षा व्यवस्था अर्थात पुस्तकों तक विद्यार्थियों को सीमित नहीं करती बल्कि इनका उद्देश्य इन आदिवासी बच्चों को अपने इतिहास, अपनी ज़मीन, अपने रीति रिवाजों,अपने संघर्ष से रूबरू करवाना है। साथ ही हस्त शिल्प एवं व्यावसायिक कौशल जैसे खेती, मूर्तिकला व टोकरी बुनाई भी सिखायी जाती है। जीवनशाला के अध्यापक -अध्यापिका ऊँची डिग्री वाले नहीं होते परन्तु वे एक- एक बच्चे का व्यक्तिगत रूप से ख्याल रखते हैं। आज बहुत सारे बच्चे आगे की पढ़ाई के लिए दूसरी जगह चले गए और कुछ जीवनशाला में ही पढ़ाने लगे। भूतपूर्व छात्र पवन और दयाल सोलंकी ऐसे ही छात्र हैं जो आज जीवनशाला में पढ़ाने के लिए वापस आए हैं।

\section{राजनीतिक अधिकारों के प्रति जागरूकता}

शुरूआत में घाटी के स्थानीय लोगों ने मेधा पाटकर और सामाजिक कार्यकताओं पर विश्वास नहीं 
किया। लेकिन धीरेथीरे समय के साथ स्थिति बदली जब वालों को उनके कानूनी हक और इन लोगों ने गाँव अधिकारों के बारे में बताया। तब इन्हें यह अहसास हुआ कि सरकार जनता से ही चलती है और सरकार से काम करवाया जा सकता है, जरूरत है तो सिर्फ जागरूक होकर संगठित रूप में सरकार की अमानवीय परियोजनाओं के खिलाफ आवाज उठाकर अपने अधिकारों को सुरक्षित रखने की।यहाँ के स्थानीय लोगों के लिए यह संघर्ष आज स्वाधीनता का संघर्ष बन गया है सरकार की अनीतियों के खिलाफ लड़ते हुए वे अपनी जान की बाजी लगाने को तैयार है। सरकार अफसर अक्सर इन गाँव वालों को रिश्वत देकर फुसलाने की कोशिश करते हैं, पर ये दृढ़ निश्चयी योद्धा उन पैसों की तरफ देखते तक नहीं। गोखरू मंगल, खड़या भादल गाँव का मुखिया कहता है-'इन पैसों से अगर मैं अपने घर का खर्च चलाऊँगा तो अन्य गाँव वालों के खून पीने के समान होगा। 8

वस्तुतः गाँववालो और आदिवासियों के प्रतिनिधियों को अपनी भूमिका का अहसास है। वे दुनिया के सामने अपनी बात भावनाओं में बहकर नही बल्कि तथ्यों और तर्को के साथ रखते हैं। सुप्रीम कोर्ट, दिल्ली के बाहर जब पत्रकारों ने मेधा पाटकर से पूछा कि क्या होगा अगर सरकार आपकी बात नही मानेगी तो? तब मेधा पाटकर ने कहा, मुझसे नहीं जो लोग इस परियोजना के बनने से प्रभावित होंगे, उनसेपूछो। तब बावा महारिया पत्रकारों से कहता हैतुम लोगों ने शायद नर्मदा घाटी नहीं देखी है। हमें अपने गाँवों में बिजली की कोई आवश्यकता नही है। हम वहाँ सम्पन्न और खुशहाल जीवन बितारहें है। फिर भी सरकार हमें भूमिहीन करके इतना बड़ा बाँध बना रही है, जिससे बिजली का उत्पादन हो। यहाँ दिल्ली में यमुना नदी है, क्यों तुम यमुना में बाँध बनाकर बिजली का उत्पादन नही करते हो क्या हमारे जीने का अधिकार दिल्लीवासियों के जीने के अधिकार से अलग है? 9

\section{जातीय समानता}

दशकों के संघर्ष के बाद जीवन ने घाटी के लोगों को कई महत्वपूर्ण पाठ सिखाया है। उन्होंने इस लम्बे
संघर्ष के दौरान यह अनुभव किया कि जातपाँत-, ऊँचनीच, छूत-अछूत से बड़ी कई और समस्याएँ है। इस जातपाँत, छूतअछूत-, सम्प्रदायिक भेदभाव से ऊपर उठकर संगठित होकर ही अपनी लड़ाई जीत सकेंगे। आंदोलन ने हमेशा ही जातीयता के आधार पर भेदभाव के व्यवहार का निषेध किया है। लोगों को साथ जोड़ने के लिए कार्यकताओं ने सभा-सम्मेलनों में साथ बैठने, रहने, खानेपीने की व्यवस्था की है। मेधा पाटकर सभाओं और धरनों की योजना बनाने के लिए जबघूमती है तो जब गाँवदलितों के घर उनके बीच रहती है, खाती है।

इस तरह की सभाओ में जब लोगों के लिए खाना बनता है तो मेधा पाटकर इस बात पर जोर देती है कि सबके लिए समान खाना बनेगा और एक ही बर्तन और चूल्हे में बनेगा। 'छोटा बाडाडा' गाँव से आने वाली कमला यादव इस संबंध में कहती है -'भेदभाव गाँवों में किया जाता था, पर आंदोलन का हिस्सा हो जाने के बाद लोग छूआछूत की भावना को भूला देते है। हमें साथ रहना, साथ खाना होता है। हमने कभी भी जातीय समानता के बारे में सभा नहीं की पर आंदोलन के कार्यक्रमों में हम उसे व्यवहार में लाते है। दीदी हमेशा यह (मेधा पाटकर) सुनिश्चित करती थी कि कोई आदिवासी मंडली के बाहर न बैठा हो, वो उन्हें बुलाती थी और अपने पास बिठाती थी। धीरे धीरे सब साथ बैठने लगे। 10

जिससे लोगों को इस बात का अहसास हुआ कि जातीय वर्गभेद की नीतियाँ, उन्हें बाकी लोगों से जुड़ने नहीं दे रही हैं और संघर्ष को कमजोर बना रही है। जातीयता की यह भावना आज भी वहाँ मौजूद है, पुरानेपीढ़ी के साथ। लेकिन आज आंदोलन के प्रयासों से पात के -समानता में विश्वास करने वाली युवा पीढ़ी जात दूसरे-इन बंधनों से मुक्त है। वे एक को अपना रहे हैं। आज लोग आदिवासियों के यहाँ शादी में जाते हैं, दलितों के साथ उठतेजाति और निम्न वर्ग के बीच -खाते है। ऊँचीप्रदान से -बातचीत और सामाजिक व्यवहार के आदान स्थिति काफी सुधरी है। लोग आज धर्म के नाम को नहीं व्यक्ति के कार्य को महत्व देते है। 
स्री सशक्तिकरण-

आंदोलन ने स्तिरायों को सशक्त बनाया है। पहले गाँव और आदिवासी स्त्रियाँ कभी घर के बाहर नही जाती थी। रैलियों में जाना, नारा लगाना, आम सभा में भाषण देना ये सब उन्होंने पहले कभी नहीं किया। शुरुआत में बूढ़ी महिलाएँ ही जवाब देती थीऔर नवयुवतियाँ घूघट काढ़े कोने में बैठी रहती थी। इन युवतियों को आगे बढ़ने के लिए प्रोत्साहित किया। आज सभी स्तियाँ आंदोलन में सक्रिय रूप से भाग लेती है।

आंदोलन में अपनी जगह और सम्मान बनाने के बाद इनकी भागीदारी और प्रतिबद्धतापुरुषों से कई गुना बेहतर साबित हुई है। जून2008 में, बडवानी के पास तलून गाँव में भूमि सत्याग्रह धरना था। पुलिस ने कुछ आदिवासी लोगों को गिरफ्तार करने की कोशिश की तब महिलाओं ने पुलिस का रास्ता रोका और पुलिस को गिरफ्तार लोगों से भरी पूरी बस को खाली करना पड़ा।11 महिलाओं की भागीदारी आंदोलन में महत्वपूर्ण रही है,। सभाओं और चर्चाओं में निर्णय लेने में उन्होंने महत्वपूर्ण भूमिका अदा की है। और सरकार को कई बार चुनौती दी है। महिलाएँ यहाँ घर का कामकाज परिवार को तो संभालती ही है साथ ही इस संघर्ष को मजबूत बनाने के लिए हर मोड़ पर आगे रहती है। आंदोलन के कार्यक्रमों में भाग लेने की प्रक्रिया में औरतों के लिए समाज द्वारा बनाए गए कई रूढ़िगत परंपराएँ जैसे पर्दाप्रथा आदि टूटती चली गई।

\section{निष्कर्ष:}

आंदोलन स्थायी रूप से बाँध के निर्माण पर रोक नहीं लगा पाया। संघर्ष अभी जारी है, पर महत्वपूर्ण बात ये है कि इस आंदोलन के चलने से घाटी के समाज में आए सकारात्मक बदलाव और ये बदलाव लोगों की समझ, उनके दृष्टिकोण और जागरूकता में देखा जा सकता है।

महिलाओं और आदिवासियों के सशक्तीकरण, जनआंदोलनों की शक्ति का प्रदर्शन और इन सबके माध्यम से दलितों के स्वर सशक्त हुए। यह संघर्ष शक्तिहीन और गरीब जनता के लिए महत्वपूर्ण है। संघर्ष करते हुए ये लोग जानते है कि अगरवो संगठित रहकर लड़ेगे, तो बहुत
कुछ किया जा सकता है। नर्मदा घाटी के लोग मेधा पाटकर का उपकार हमेशा याद रखेंगे, क्योंकि उन्हें मालूम है इस घाटी को बचाने में बीते वर्षों में जितना काम पाटकर ने अपने दृढ़ इच्छाशक्ति से किया, उतना काम बहुत से आदमी भी मिलकर नही कर सके। क्योंकि एक स्री की शक्ति अद्वितीय और मजबूत होती है। स्त्रियों की मानसिक शक्ति पुरुषों के शरीरिक बल की तुलना में काफी मजबूत होती है। इसके बावजूद इसमें कोई दो राय नही है कि यहाँ के स्थानीय लोगों की दृढ़ इच्छाशक्ति, जिजीविषा, एवं संगठित शक्ति ने इस आंदोलन को आज इस मुकाम पर पहुँचाया है। सरकार के खिलाफ डूब के क्षेत्रों में चढ़ते हुए पानी के अन्दर खड़े ये नारे लगाते है 'डूबेगे पर हटेंगे नहीं ।

आंदोलन यहाँ के लोगों की जिन्दगी बन गई है। इन्हें आशा है कि अगर ये संगठित होकर लड़ेगे तो सारी समस्याओं का निदान हो सकता है, चाहे वो कोई भी समस्या होबाँध-, भूमि, आजीविका आदि। सरकार ने कुछ लोगों को भूमि दी पर उन्होंने लेने से इंकार कर दिया। बाबा महारिया उन लोगों में से एक है। वो कहता है, 'मैंने सरकार को यह कह कर चुनौती दी कि मै पुनर्वास स्वीकार करने वाला आखरी व्यक्ति होऊँगा। मैं यहाँ रहूँगा जब तक कि सभी लोग, सभी गाँवों, सभी घरों को व्यवस्थित रूप से पुर्नवास न कर दिया जाए। सरकार ने मेरे सामने भूमि का प्रस्ताव रखा है पर मैं यहाँ जलसिंघ ) से नहीं हटूंगा। जब तक (गाँव सभी को उपजाऊ भूमि मिल जाए। 12

लगातार डूब की स्थिति और विस्थापित की जिन्दगी जीते हुए भी इनका जुझारूपन चुका नहीं है बल्कि और बढ़ गया है। कवयित्री रजनी अनुरागी की निम्नलिखित पंक्तियाँ घाटी के निवासियों की भावनाओं को पूर्णरूप से व्यक्त करतीहै-

"मिश्र के जन सैलाब के आगे नतमस्तक है हुस्ती मुबारक भ्रष्टाचार, मंहगाई और बेरोजगारी से त्रस्त जनता कब तक सहे

चुप्पी बेबसी नहीं होती 
उसके पीछे चल रहा होता है गहरा विचार मंथन दिमाग की हांडी में परिपक्क हो रहे होते हैं विचार बेखौफ उतरने लगती है जब जनता सड़कों पर

एक से एक ग्यारह और ग्यारह से ग्यारह, ग्यारह लाख हो जाते हैं

आँखों से शोषण का अंधेरा छंट जाता है

तनने लगती है मुट्टियाँ और उठती हैं एक साथ

जनता जूझती है निराहार, निरस्त्र सशस्त्र सेनाओं से

मजबूत कलेजे वाले, हर तूफान से लेते है लोहाबिना लोहे के

एक कठिन दौर से गुजरते लोग जान जाते हैं

कि टालने से टलती नहीं चीजें, बिगड़ जाती हैं

पुर्नस्थापित होते हैं वे तमाम मूल्य, जिनके लिए है वे संघर्षरत

कितने ही हुस्री मुबारक बैठे हैं, अलजीरिया, सीरिया और लीबिया में

और हमारे आस पास भी कब्जा किए हमारे दिलो दिमाग पर

तहरीर चौक की जनचेतना देती है संदेश

लोकतंत्र के नाम पर जो भी बन बैठेगा तानाशाह

ये मजबूत जनता उसे ऐसे ही कर देगी मजबूर"13

\section{संदर्भ ग्रंथ सूची}

1. https://m.economictimes.com/hindi/wealth/ personal-finance/why-new-agriculturallaws-are-not-just-problems-offarmers/articleshow $/ .79866861 \mathrm{cms}$

2. अमर उजाला, हरियाणा, झांझर -बहादुरगढ़, ई-पेपर 19जनवरी2021

https://www.amarujala.com/haryana/jhajjar -bahadurgarh/ration-system-will-bedestroyed-by-new-agricultural-law-medhapatkar-bahadurgarh-news-rtk5914877191
3. मेधा पाटकर से आलोक प्रकाश पुतुल की बातचीत"हमारी बात नहीं मानेंगे तो पछताना पड़ेगा" रविवारकॉम., 12.01.2010

https://hindi.indiawaterportal.org/content/h amaarai-baata-nahain-maanaengae-taopachataanaa-padaegaa/content-typepage/29777

4. वही

5. वही

6. Plural Narratives from Narmada Valley, Delhi Solidarity Group Oct 2010, Page 1011

7. Narmada Jeevan Shalas Bring Hope for Children Affected by the sardar sarovar project https://www.videovolunteers.org/narmadajeevan-shalas-bring-hope-for-childrenaffected- by-the-sardar-sarovarproject

8. गोखरू मंगल, प्लूरल नरेटि फ्रॉम नर्मदा वैली, पृष्ठ-15

9. बाबा महारिया, प्लूरल नरेटिव फ्रॉम नर्मदा वैली, पृष्ठ-69

10. कमला यादव, प्लूरल नरैटिवस् फ्रॉम नर्मदा वैली, पृष्ठ 134

11. प्लूरल नरेटिव फ्रॉम नर्मदा वैली,पृष्ठ-43

12. बाबा महारियाए, प्लूरल नरेटिक्स् फ्राम नर्मदा वैली, पृष्ठ-66

13. रजनी अनुरागी, कविता शीर्षक - जनज्वार, कवितासंग्रह- बिना किसी भूमिका के, आरोही प्रकाशन, नई दिल्ली 